Proceedings of the LIV Zakopane School of Physics, Breaking Frontiers, Zakopane, Poland, May 21-25, 2019

\title{
Kinetics of Oxidation and Antioxidation Processes in Biological Solutions Observed by NMR Relaxation
}

\author{
D. WierzuchowskA ${ }^{a, *}$, M. WiteK $^{b}$ AND B. BlicharskA ${ }^{c}$ \\ ${ }^{a}$ Institute of Physics, Pedagogical University, Podchorążych 2, 30-084 Kraków, Poland \\ ${ }^{b}$ Department of Refrigeration and Food Concentrates, Faculty of Food Technology, \\ University of Agriculture in Krakow, Balicka 122, 30-149 Kraków, Poland \\ 'Institute of Physics, Jagiellonian University, S. Łojasiewicza 11, 30-348 Kraków, Poland
}

\begin{abstract}
Preliminary studies on the application of NMR relaxation measurements for observations of the kinetics of oxidation processes in tea samples were performed. Tea is a rich source of flavonoids and other compounds with great antioxidant capacity and is therefore beneficial for human health. Its antioxidant potential depends on the type of tea and the method of preparation. Green and black tea prepared from tea bags brewed in water at $90{ }^{\circ} \mathrm{C}$ for 20 and 60 min were examined. Oxidation processes were induced in tea samples by adding $3 \% \mathrm{H}_{2} \mathrm{O}_{2}$ in 1:10 proportion. Observed time courses of spin-lattice $T_{1}$ relaxation time and parameters obtained from fitting of combined exponential and compressed-exponential function, depended on kind of tea, brewing time, and milk addition. The obtained results showed the usefulness of the NMR relaxation measurements for observation of oxidation processes and antioxidant activity.
\end{abstract}

DOI: 10.12693/APhysPolA.137.21

PACS/topics: NMR relaxation, tea, oxidation processes, antioxidants' activity

\section{Introduction}

Tea, one of the most popular beverage in the world, is regarded to have many benefits on human health, e.g., prevention from cancer, cardiovascular, neurodegenerative diseases and others. Tea infusions contain catechins, theaflavins, oxiaromatic acids, flavonols, tannins, etc., which can act, in vivo and in vitro, either as proor antioxidants. They can act as antioxidants directly scavenging free radicals or chelating transitional metals, but on the other hand, they can lead to free radicals' formation. Each tea is different in terms of composition and concentration of antioxidant compounds thus may exert different benefits on human health. Also the manner of brewing tea may influence activity of antioxidants. Many researches, involving different physical and chemical methods, are provided to find out the best kind of tea and manner of preparation $[1,2]$.

The antioxidant potential of tea is usually assessed by spectrophotometric methods as free radical scavenging method (DPPH or ABTS) or by measuring the reduction of oxidation state of some metal ions by antioxidants (FRAP or CUPRAC methods) [3]. In this communication the NMR relaxation method for observation of oxidation and antioxidation processes was proposed and tested.

\footnotetext{
* corresponding author; e-mail: dorota.wierzuchowska@up.krakow.pl
}

\section{Materials and methods}

Black tea in $1.6 \mathrm{~g}$ tea bags and green tea in $1.75 \mathrm{~g}$ bags, commercially available were used to prepare tea infusion samples with $200 \mathrm{ml}$ of tap water at $90^{\circ} \mathrm{C}$ and brewing time of 20 and 60 min. Fresh cow milk was added in 1:10 proportion to black tea infusions.

The 3\% hydrogen peroxide (purchased at pharmacy) as an initiator of oxidation process was added to tea infusions in 1:10 proportion and well mixed with investigated solution immediately before measurements.

Proton NMR relaxation time $T_{1}$ was measured using a Minispec Bruker spectrometer, operating at $1.4 \mathrm{~T}$ $(60 \mathrm{MHz})$ at stabilized room temperature $\left(25^{\circ} \mathrm{C}\right)$, using an inversion recovery (IR) method. The values of $\tau$ at IR sequence ranged from 50 to $20000 \mathrm{~ms}$. The $90^{\circ}$ pulse length was $1.3 \mu \mathrm{s}$, and the repetition time between scans was at least 5 times longer than $T_{1}$.

Data were analyzed using a mono-exponential function by means of the standard Bruker procedure. Measurements errors were $2 \%$.

\section{Results and discussion}

Water proton spin-lattice relaxation time $T_{1}$ as measured at $25^{\circ} \mathrm{C}$ in distilled water after addition of $3 \% \mathrm{H}_{2} \mathrm{O}_{2}$ in 1:10 proportion remains unchanged because of a lack of species which could be oxidized. However, after addition of hydrogen peroxide to drinking water, reactive oxygen species are successively produced. They are paramagnetic and cause exponential drop of $T_{1}$. Time courses of $T_{1}$ which illustrate kinetics of oxidation processes in distilled and drinking water are presented in Fig. 1. 


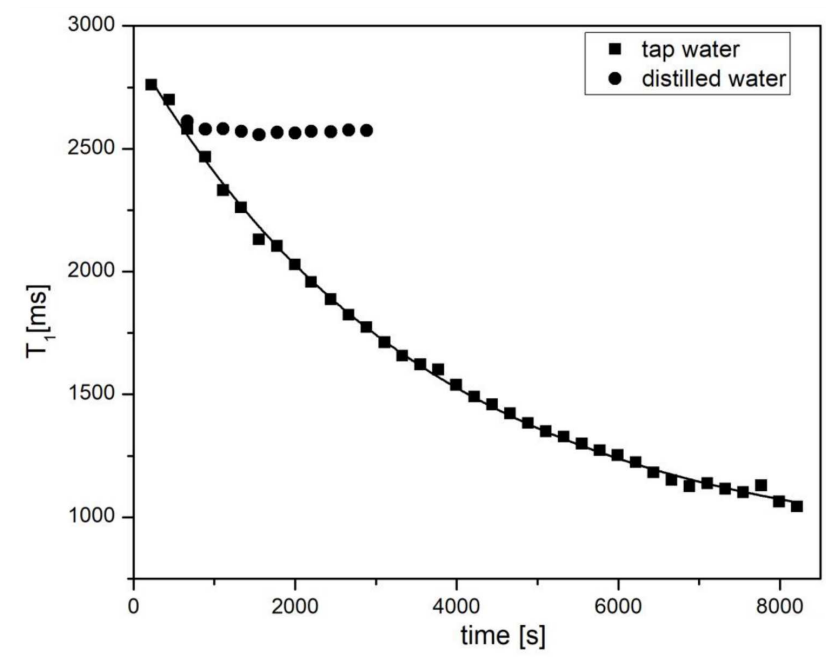

Fig. 1. The time courses of $T_{1}$ relaxation time after adding $3 \% \mathrm{H}_{2} \mathrm{O}_{2}$ in a ratio of $1: 10$ to distilled and drinking water. $T_{1}$ values before adding $\mathrm{H}_{2} \mathrm{O}_{2}$ were $2957 \pm 8 \mathrm{~ms}$ and $2920 \pm 10 \mathrm{~ms}$, respectively.

If drinking water was used to prepare tea extracts, the $T_{1}$ run in time is different. Figure 2 shows time courses of $T_{1}$ obtained after addition of $3 \% \mathrm{H}_{2} \mathrm{O}_{2}$ to black tea samples taken after $20 \mathrm{~min}$ and $60 \mathrm{~min}$ of brewing, as well as a green tea sample after $20 \mathrm{~min}$ of brewing. The time course of $T_{1}$ for black tea infusion (20 min) is initially an exponential decay, thus shortening of $T_{1}$ indicates oxidation processes. After reaching the minimum, $T_{1}$ starts to increase as a result of antioxidants' activity. This curve is similar to the time course for protein solutions with the addition of vitamin C [4] and may be interpreted as an action of antioxidants present in tea. In aqueous solutions of proteins

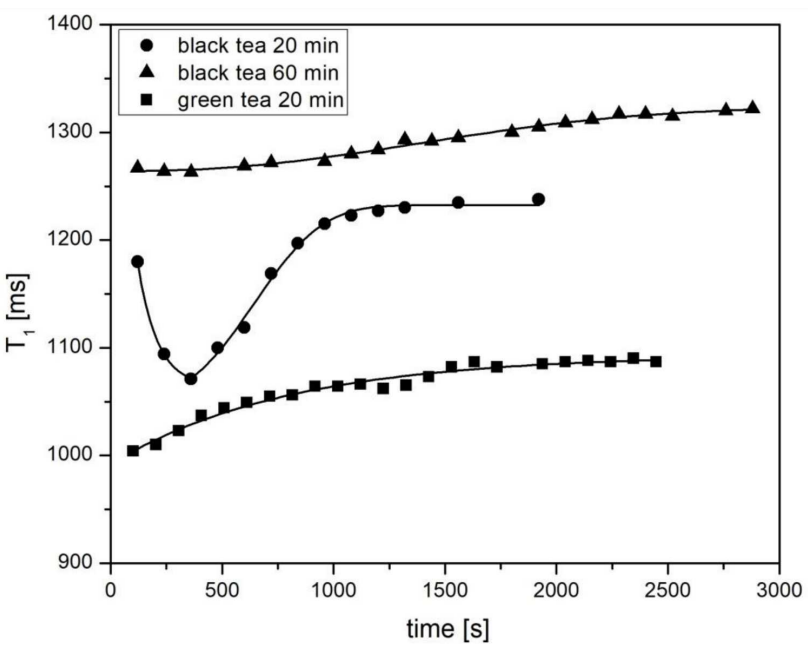

Fig. 2. The time courses of $T_{1}$ relaxation time after adding $3 \% \mathrm{H}_{2} \mathrm{O}_{2}$ to black tea after brewing at $90^{\circ} \mathrm{C}$ for $20 \mathrm{~min}$ and $60 \mathrm{~min}$ and to green tea after $20 \mathrm{~min}$ of brewing. $T_{1}$ values before adding of $\mathrm{H}_{2} \mathrm{O}_{2}$ were $1184 \pm 5 \mathrm{~ms}$, $1018 \pm 6 \mathrm{~ms}$ and $792 \pm 5 \mathrm{~ms}$, respectively.

(like egg white or bovine serum albumin) the time courses, obtained after the addition of hydrogen peroxide, were well fitted by exponential decays with parameters depending on the type and concentration of proteins. Adding an antioxidant (e.g., ascorbic acid or glutathione) to protein solutions resulted in a different behavior - after exponential decay and reaching a minimum - an increase of the relaxation times was observed [4]. Similar time courses were measured also in native blood serums which contained the endogenous antioxidants [5].

The time courses of $T_{1}$ were well fitted by combining the exponential and compressed-exponential function according to the formula:

$$
T_{1}= \begin{cases}T_{1,0}+T_{d} \exp \left(-\frac{t-t_{c}}{t_{d}}\right) & \text { for } t<t_{c} \\ T_{1,0}+T_{d}+T_{g}\left(\exp \left(-\left(\frac{t_{c}}{t_{g}}\right)^{n}\right)-\exp \left(-\left(\frac{t}{t_{g}}\right)^{n}\right)\right) & \text { for } t>t_{c}\end{cases}
$$

where $2 \leq \mathrm{n}<4, T_{1,0}$ is equilibrium value of $T_{1}$ relaxation time, $T_{d}$ and $T_{g}$ are the loss and gain in value of $T_{1}, t_{d}$ and $t_{g}$ are time constants, $t_{c}$ is turning time constant. Values of selected parameters of time courses obtained by fitting data presented in Fig. 2 are shown in Table I.

Kinetics of free radical scavenging processes in extracts derived from plants, among others in tea, measured by spectrophotometric methods, are usually described by exponential functions [6]. NMR relaxation time of water is affected by a series of interactions, which are: strong interaction of water with free radicals (paramagnetic ions) that shorten $T_{1}$ value (the first term in formula (1)) and interaction with radicals quenched by antioxidants and with antioxidants themselves (second term in formula (1)). The first described process may be very fast and thus not measurable at all (Fig. 2 and Table I). The second process, due to variety and amount of antioxidant compounds present in tea infusions [3], may result from a series of higher-order chemical reactions [7], and hence this, unusual compressed-exponential relationship $(n>1)$ was proposed. This two-step relationship was only observed for the tea brewed for $20 \mathrm{~min}$.

Time courses of $T_{1}$ changed if the time of brewing of black tea was longer $(60 \mathrm{~min})$ or when green tea was used (Fig. 2). There was no clear decay and no clear minimum 
TABLE I

Selected parameters of time courses presented in Fig. 2

\begin{tabular}{l|c|c|c|c|c|c}
\hline \hline & $T_{d}[\mathrm{~ms}]$ & $t_{d}[\mathrm{~s}]$ & $t_{c}[\mathrm{~s}]$ & $n$ & $T_{g}[\mathrm{~ms}]$ & $t_{g}[\mathrm{~s}]$ \\
\hline black tea 20 min & 8.0 & 89.4 & 359 & 3.1 & 179.4 & 729 \\
black tea 60 min & 0 & - & - & 2.5 & 59.5 & 1763 \\
green tea 20 min & 0 & - & - & 1 & 101.3 & 805
\end{tabular}

for them and only the second part in formula (1) was used as a match to the data (Table I). For green tea time course only an exponential increase of $T_{1}$ was observed ( $n=1$ and $t_{c}=0$ and $T_{d}=0$ ), which may suggest that because of a greater antioxidant capacity of this green tea in comparison to black tea for the same brewing time, an antioxidant action is faster. Therefore, a characteristic decrease (exponential decay) in $T_{1}$, which corresponds to the oxidation process, was not observed after the addition of $\mathrm{H}_{2} \mathrm{O}_{2}$ during the experimental time.

Apparently, extending the time of brewing of black tea also causes that only an increase in the time course of $T_{1}$ was observed over time. The nature of changes and fitted parameters are also different (Table I). They may suggest faster antioxidant action immediately after adding of hydrogen peroxide in comparison to tea brewed for $20 \mathrm{~min}$. The shortest $t_{g}$ time and the largest increase in $T_{g}$ parameter were observed for black tea brewed for 20 min as compared to other samples (Table I). It may indicate that this tea contained antioxidants with high efficiency with respect to the green tea. However, their effect was significantly reduced when the brewing time increased to $60 \min \left(T_{g}\right.$ - lower, $t_{g}$ - higher $)$, which was probably associated with the process of degradation of bioactive substances.

Moreover, the addition of milk to the infusions of black tea caused a disturbance of the regular time course of $T_{1}$ (data not shown). Therefore, it was not possible to match any functional dependence to these measurements. This is probably related to the fact, known in the literature, that milk inhibits anti-oxidation processes [8].

\section{Conclusions}

The presented results and earlier made measurements of $T_{1}$ time courses in blood serum of various origins indicate the possibilities of using the NMR relaxation method to study the kinetics of oxidation and anti-oxidation processes in biological samples. It can be seen that regardless of the type of sample, if it contains any antioxidant compounds, the addition of $\mathrm{H}_{2} \mathrm{O}_{2}$ induces a specific kinetics of $T_{1}$, the course which most likely depends on the type and amount of antioxidant. Changes in $T_{1}$ time courses, described by the proposed model, showed both the effect of free radical formation and the oxidation process (shortening of $T_{1}$ in time) and the antioxidant activity of bioactive compounds in tea extracts (increase of $T_{1}$ in time). The parameters of the $T_{1}$ kinetics model allowed to assess oxidation rates and the effectiveness of antioxidants. Thus, the proposed NMR relaxation method can be useful to study the nature of oxidation and antioxidant processes.

\section{References}

[1] A. Yashin, Y. Yashin, B. Nemzer, Am. J. Biomed. Sci. 3, 322 (2011).

[2] S.P.J. Namal Senanayake, J. Function. Foods 5, 1529 (2013).

[3] I. Peluso, M. Serafini, Brit. J. Pharmacol. 174, 1195 (2017).

[4] D. Wierzuchowska, L.W. Skorski, B. Blicharska, Acta Phys. Pol. A 129, 226 (2016).

[5] D. Wierzuchowska, M. Witek, B. Blicharska, Acta Phys. Pol. A 133, 289 (2018).

[6] A. Fadda, M. Serra, M.G. Molinu, E. Azara, A. Barberis, D. Sanna, J. Food Composit. Anal. 35, 112 (2014).

[7] K.Y. Luna-Ramirez, S. Arellano-Cardenas, S. GarciaPinilla, M. Comejo-Mazon, Rev. Mexic. Ingen. Quim. 16, 121 (2017).

[8] S.C. Langley-Evans, Int. J. Food Sci. Nutr. 51, 181 (2000). 